\title{
DEVELOPMENTAL DEFECTS OF THE CISTERNA MAGNA AND DURA MATER
}

\author{
BY \\ E. GRAEME ROBERTSON
}

\section{From the Department of Neurology and Neurosurgery, Royal Melbourne Hospital, Melbourne, Australia}

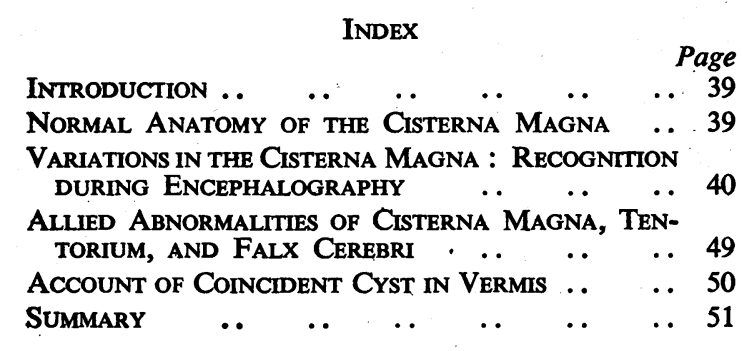

\section{Introduction}

Cerebral defects of developmental origin, apart from those involving the nervous parenchyma, are to be found chiefly in the neighbourhood of the roof of the third ventricle. The complex developmental evolution of the structures in this region may predispose to imperfect development, whether the cause be some variety of damage which impairs development, or some genetically transmitted factor.

The suprapineal recess varies considerably in size, and in ten of five hundred cases it was found to be much larger than usual. The two leaves which fuse to form the septum pellucidum sometimes remain separated and a fluid-filled cavity separates the lateral ventricles. Sometimes one or both walls of this cavity are fenestrated, thus allowing communication between the lateral ventricle and the cavum septi pellucidi. The arachnoid-lined space in the velum interpositum is obliterated during normal development, but sometimes persists into adult life. Finally, the corpus callosum and fornix may be absent or may cease to develop at an early stage.

The cisterna magna also is subject to considerable variation in size and shape, due to causes active during development. The shape and extent of the cisterna magna are important in the performance of encephalography, and the recognition of abnórmality is important if ventricular filling is to be secured in the patient in whom an unusual state exists. The lack of recognition of the anomalies to be described is due to the difficulty of delineating the cistern in specimens, for without floating the arachnoid away from the brain it is difficult to delimit the periphery of the cistern. - It is only when gas in the cistern clearly delimits it that these variations can be recognized.

\section{Normal Anatomy of the Cisterna Magna}

As the arachnoid membrane passes upwards from the spinal canal through the foramen magnum to enclose the contents of the posterior cranial fossa it becomes more extensive. Anteriorly the subarachnoid space suffers no interruption in front of the medulla and pons. Thus, during encephalography, gas is able to pass upwards to reach the interpeduncular cisterns and thence the cerebral subarachnoid space (Fig. 1). Posteriorly and laterally, however, the arachnoid membrane is closely applied to the pia mater on the inferior surface of the cerebellum. Thus, in this direction, the upward passage of gas is blocked (Figs. 1 and 13a). The attachment over the vermis in the median plane posteriorly is usually at a higher level than the attachment to the laterally placed hemispheres. In front, the close apposition of pia and arachnoid ceases at the ventral border of the cerebellum, a wide subarachnoid space which is continuous with that over the anterior surfaces of the medulla and pons being found in the cerebellopontine angles.

The term cisterna magna* is applied to the portion of the wide subarachnoid space below the inferior surface of the cerebellum, behind the medulla, and above the dura and arachnoid over the lower and medial part of the occipital bone. The foramen of Magendie lies at the apex of the anterior part of the cistern, at a higher level than the lower surface of the vermis. The normal disposition in this region is responsible for the filling of the ventricles when gas is introduced with the head in a flexed

* In the original description, the cisterna magna cerebellomedullaris includes that part of the subarachnoid space anterior and lateral to the medulla. However, it facilitates description, and is common usage, to apply the term cisterna magna only to that is common usage, to apply the term cisterna magna only to that
part behind the medulla. The anterior part may well be called the medullary cistern. 
position. The gas rises behind the cervical spinal cord and then through the dorsal part of the foramen magnum to the cisterna magna. It passes to the upper part of the cistern, and when the gas-fluid level sinks below the lowest part of the vermis gas will flow forwards and upwards, between the cerebellar tonsils, to the foramen of Magendie and thus into the fourth ventricle. Gas in this position is seen clearly in lateral views (Fig. 1). In axial views (films taken with the central ray directed, with varying inclination, along the median plane of the skull) it is seen as a dense, narrow shadow in the mid-line, widening below into the shadow of the remainder of the cisterna magna and above into that of the fourth ventricle. Some gas rises directly to the anterior part of the cistern, anterior to the sill provided by the vermis, and thus to the foramen of Magendie, the amount so doing increasing as the head becomes more erect.

Variations in the Cisterna Magna :

Recognition during Encephalography

The extent of the cistern over the inferior surface of the cerebellum varies considerably. In the majority of cases the cistern extends about $2.5 \mathrm{~cm}$. above the foramen magnum along the inner table of the occipital bone, reaching a point midway between the posterior lip of the foramen magnum and the internal occipital protuberance (Fig. 1). The depth (that is, the axial extent) is usually $5 \mathrm{~mm}$. The cistern is sometimes small, sometimes considerably larger. Only when the cistern is large is it well seen in axial views. Owing to its position over the lower part of the occipital table, the lateral extent of the shadow is best seen in occipito-vertical views taken with the head well flexed before gas has entered the ventricles or anterior subarachnoid spaces. The width is very variable. Sometimes the shadow is about $2 \mathrm{~cm}$. wide, sometimes much broader. The dense median shadow of the inferior occipital crest often divides the shadow into two more or less symmetrical halves (Fig. 2).

The cistern was found to extend as high as the internal occipital protuberance four times in one hundred cases (Fig. 3). In these cases the depth and the width of the cistern were usually larger than normal. Such a cistern causes a dense shadow during encephalography, owing to the large volume of gas which it contains. This may lead to confusion in interpretation of encephalograms unless the significance of the shadow is recognized. Such a large cistern may, by its capacity, interfere with the early filling of the ventricles. Thus 10 or 12 c.cm. of gas may be introduced before any passes into the ventricles. Gas begins to enter the fourth ventricle when the dorsal part of the cistern is full, but ventricular filling may be secured at an earlier stage by dorsiflexing the head. The cistern is thwos brought to a lower level than the foramen gf Magendie, and gas is decanted into the fourth ventricle.

In some cases the lateral limits of the cistern are unduly wide and are placed at a higher level ther the foramen of Magendie. Gas entering dorsalfy will not be directed to the foramen of Magendre but will flow forwards at a higher level to the cerebello-pontine angles and thus into the pontiva cisterns. This is a commonest cause of failure fill the ventricles during encephalography. Unles a special manœuvre is employed, gas will not the enter the ventricles during encephalography. The head is fully flexed during periods of introduction of gas and slowly dorsiflexed between such injections. In such full flexion the foramen of Magendie 8 higher than the point on each side at which the cisterna magna decants into the lateral recesses the medullary and pontine cisterns.

Secondly, the pia and arachnoid may not fuse over the inferior surface of the cerebellum, hences the usual barrier at the upper level of the cisternia magna does not exist (Figs. 4 and 13c). entering dorsally passes cranially around the

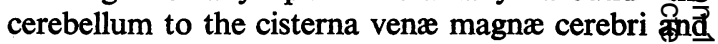
thus to the cerebral subarachnoid space. In norgath cases the subarachnoid space over the superos surface of the cerebellum may fill with gas from oh cisterna venæ magnæ, but no gas passes from there down to the inferior surface of the cerebellum lower level (Fig. 5). When the cisterna magna not closed above, gas reaches the superior surface of the cerebellum without passing along the ventra and ambient cisterns (Fig. 4). In this case, the gas is not directed to the foramen of Magendie, hence ventricular filling is difficult to secure. If the hea be kept erect, instead of flexed, some gas rises directly to the foramen of Magendie and enters th $\overrightarrow{\vec{\theta}}$ ventricles.

The anomaly which is the subject of this paper was first seen four years ago. The cisterna magna. was found to extend above the inion, almost to the apex of the occipital bone (Fig. 6a). Thus, its ape was considerably above the usual level of the tentorium. In addition to extending unusually. high, the cistern was also very wide in its lowep part (Fig. 6b). Fifteen c.cm. of gas were introduced without the appearance of ventricular shadows? By dorsiflexing the head the gas could be decanted through the foramen of Magendie into the ventricu을. lar system. Some three years later a second instancô was found during encephalography (Fig. 7), and $a^{\circ}$ year later a third exactly similar appearance (Fig. 8 S was found. In the last instance it was possible tow 
TG. 1. Normal cisterna magna in lateral view. The uninterrupted upward passage of gas anterior to the spinal cord, medulla, and pons is shown in contrast to the limitation behind by the close apposition of arachnoid and pia over the cerebellum. Gas may escape from the cisterna magna by passage $(a)$ forwards and upwards to the fourth ventricle through the anterior part of the cisterna magna (seen as a dense column leading up to the fourth ventricle), or (b) forwards at a lower level into the medullary cistern.

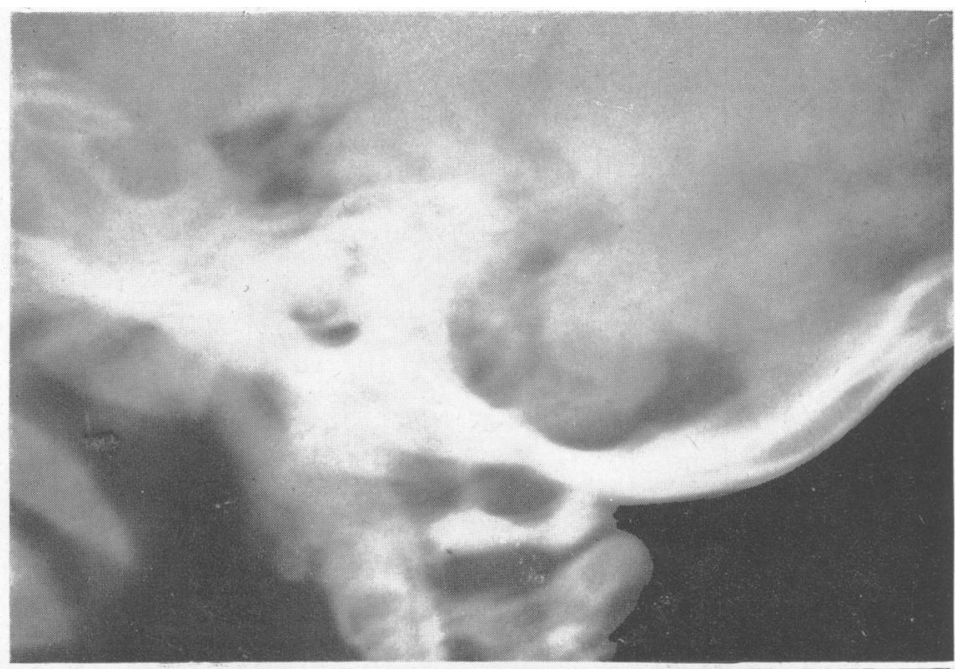

IG. 2.-Large cisterna magna in an occipito - vertical film at an early stage of encephalography. The median shadow of the anterior part of the cistern is seen.

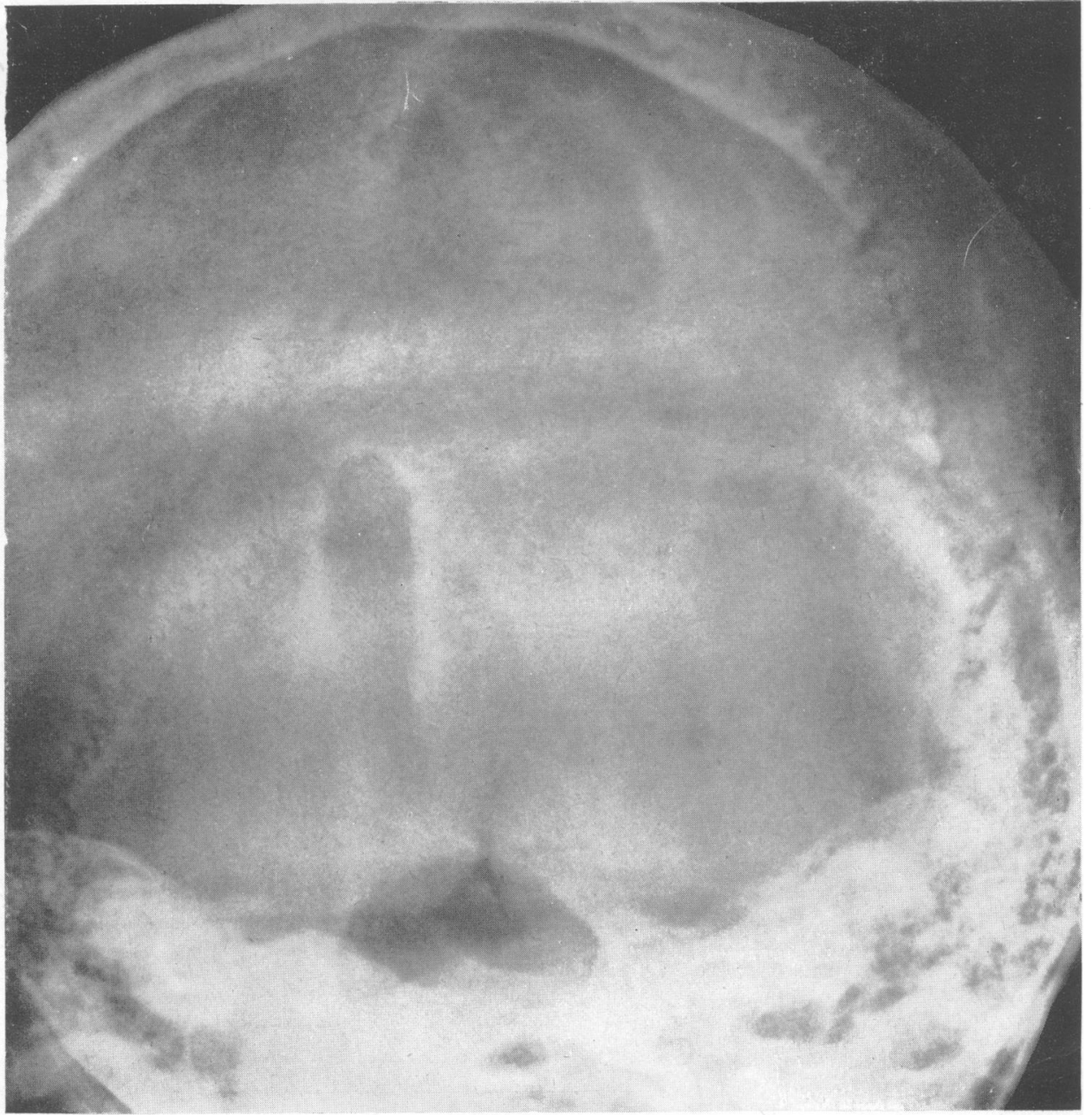




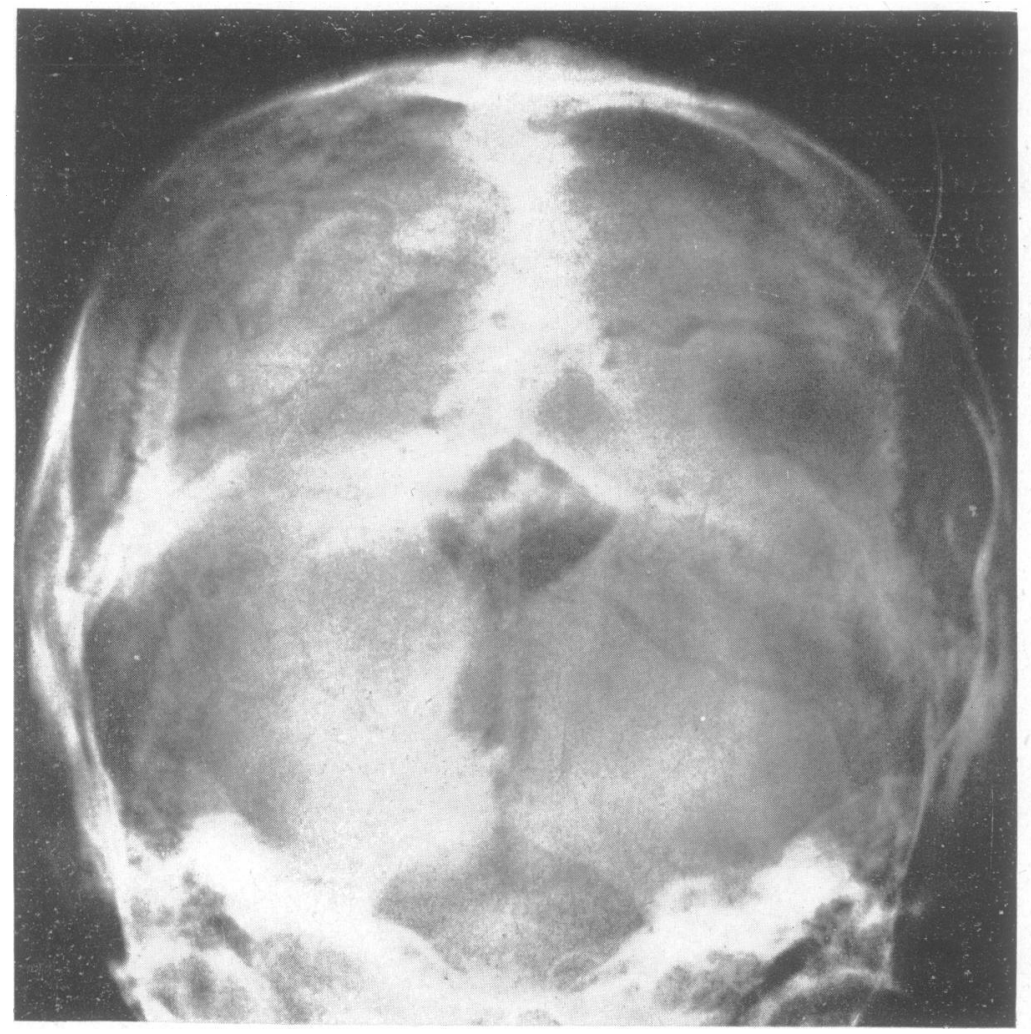

FIG. 3.-Large cisterna magna reaching the inion.

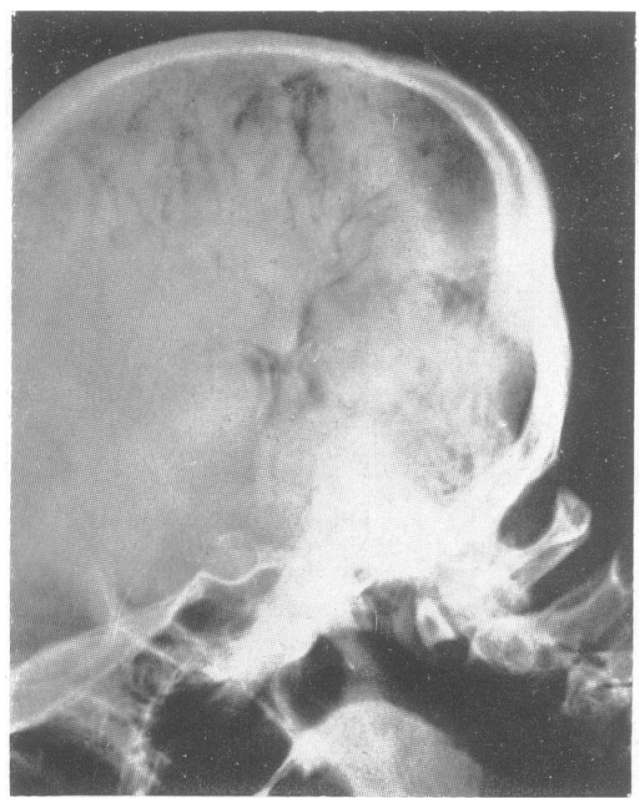

FIG. 4.-Defective occlusion of the upper limit of the cisterna magna, allowing gas to pass frecly over the cerebellum to the cisterna vena magna cerebri. 


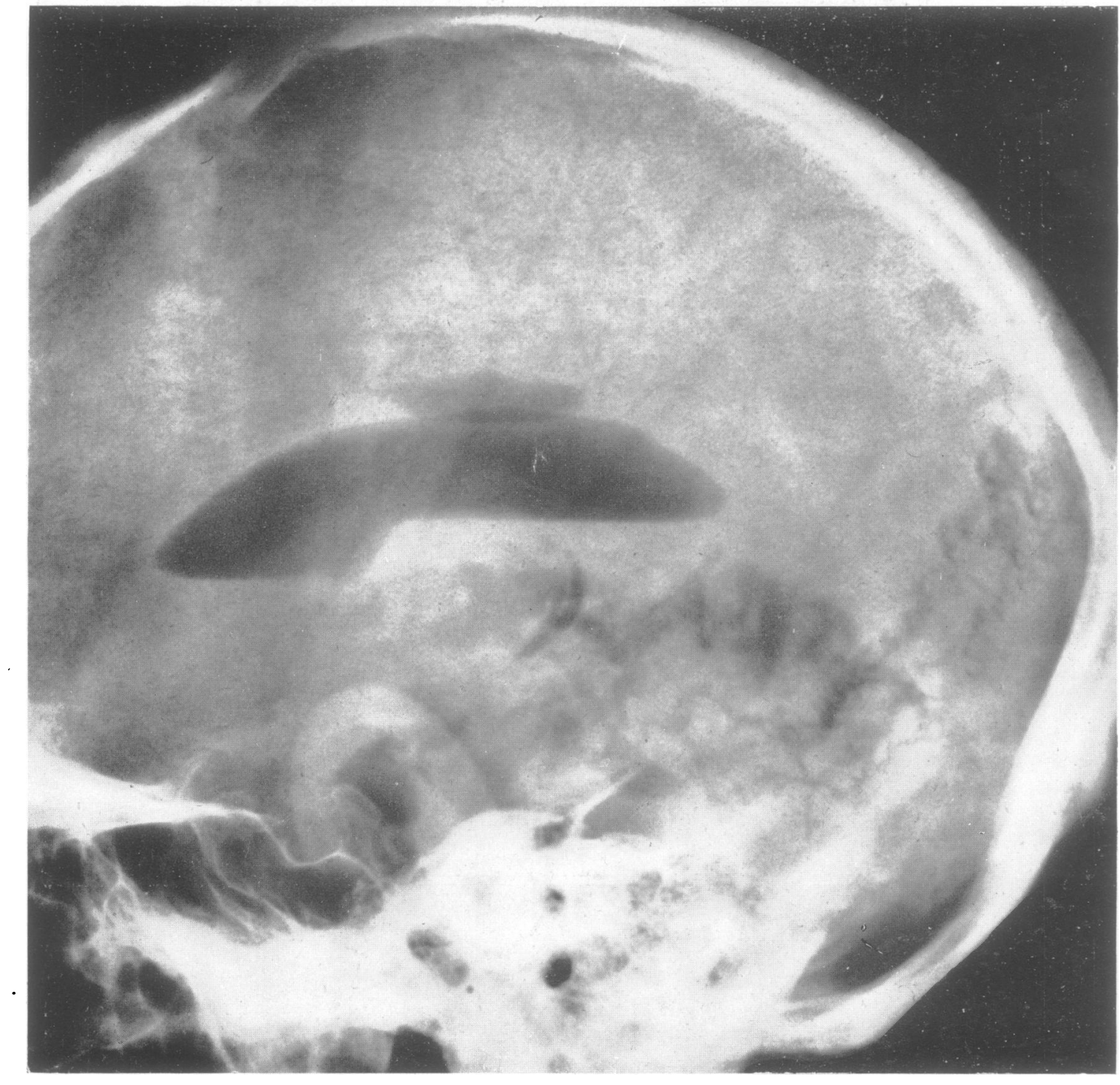

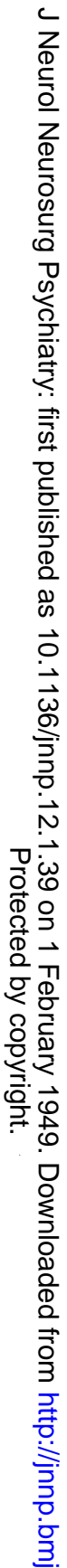

Фి

(1)

ᄋ̊

FIG. 5.-Filling of subarachnoid space over superior surface of cerebellum from the cisterna venie magna cerebri. 
影咆

훈

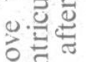

으

임듀.

는

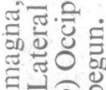

$7-20$

호 플

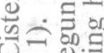

i.

o워즞

它
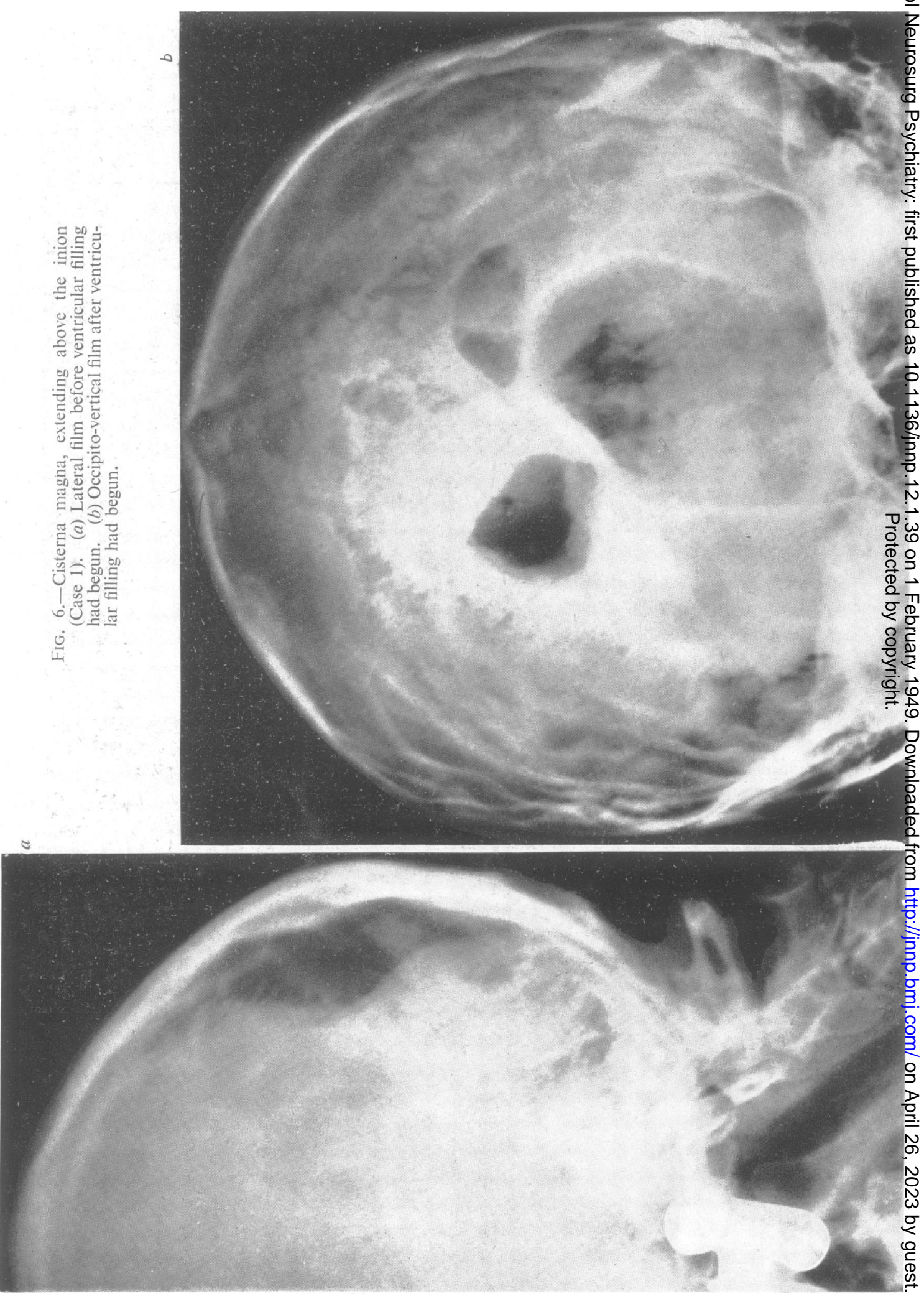


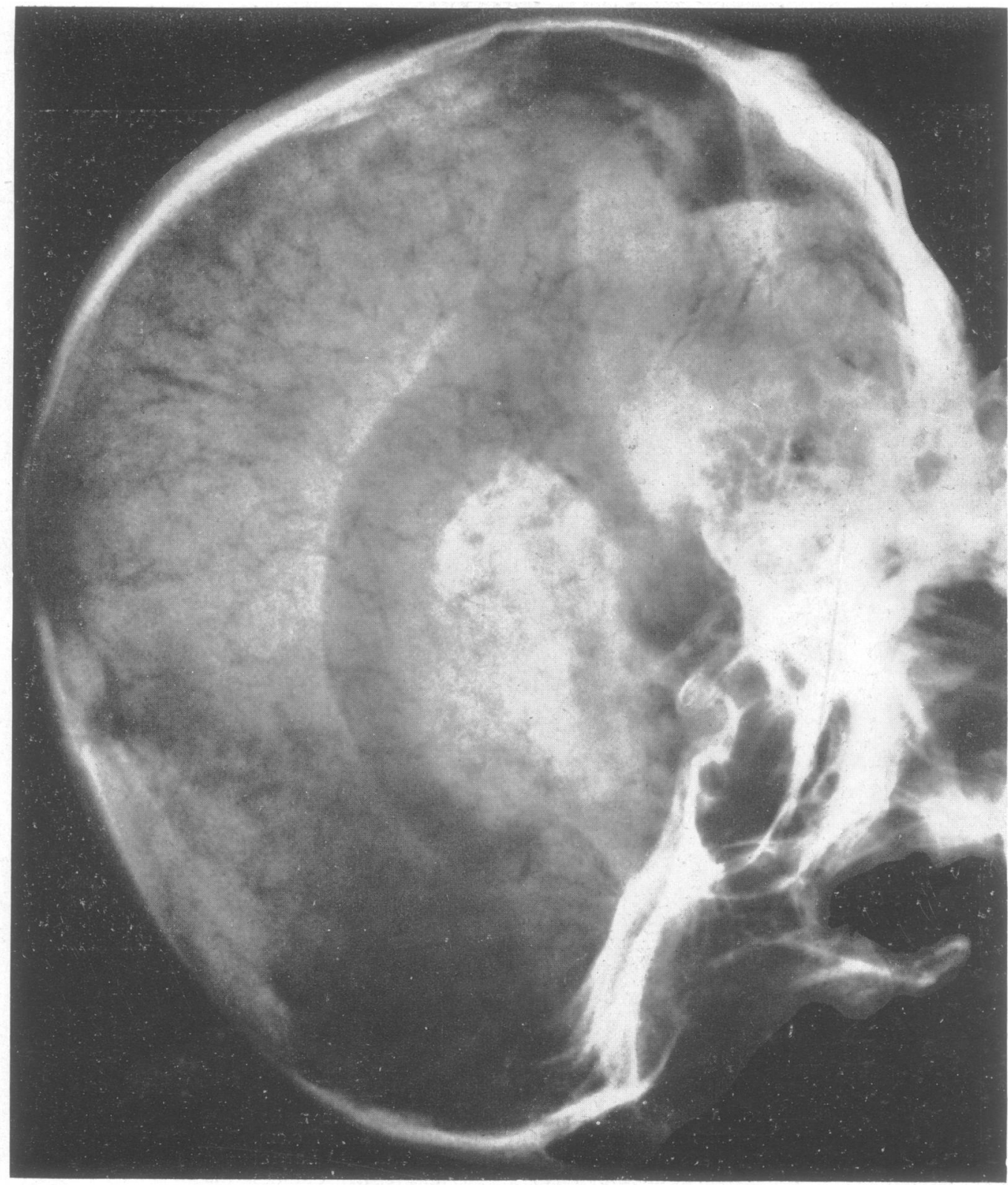

过

윽

$\stackrel{\mathbb{0}}{=}$

o

ד

$\stackrel{\infty}{\frac{\infty}{3}}$

$\stackrel{\bar{m}}{\vec{z}}$

$\stackrel{\vec{F}}{\mathrm{~s}}$

믐

$\frac{\bar{s}}{\overline{0}}$

$\frac{2}{80}$

$\stackrel{\infty}{\overrightarrow{0}}$

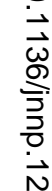

$\nabla \vec{\omega}$

o

هㅇำ

ब.

एक्ष 8 궁

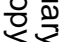

के

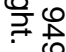

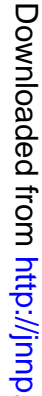

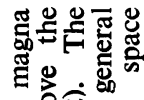

ฐ

000

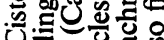

1

的.

迹 


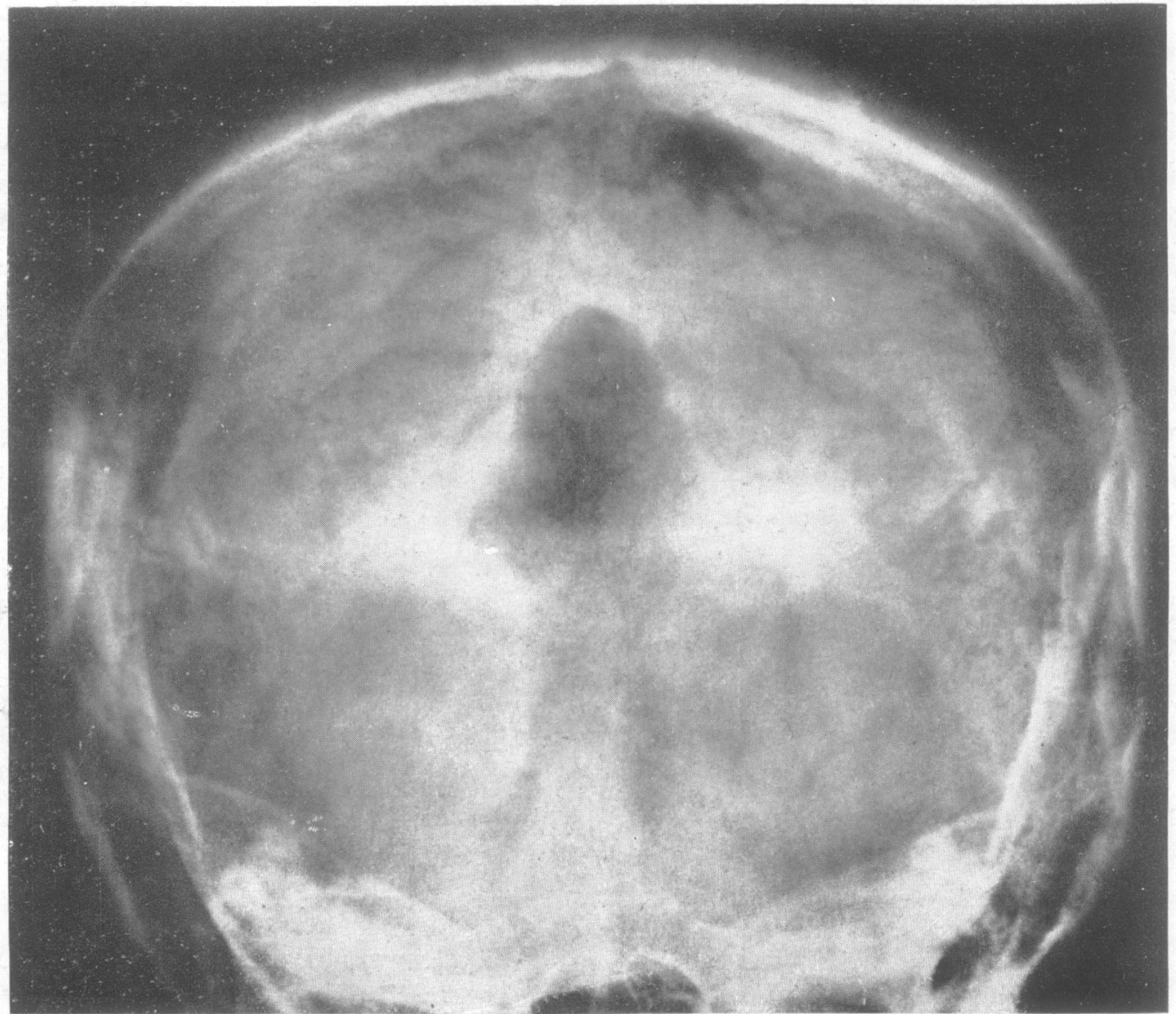

FIG. $8 a$

Fig. 8.-Cisterna magna extending higher than the inion (Case 3). (a) Occipito-vertical film, $7 \mathrm{c.cm}$. of gas outlining the apex of the cistern. (b) Lateral film at the end of filling. The large cistern outlines the vermis of the ceicellum, and (below) a cyst presenting between the cerebellar tonsils. The small interpeduncular cistern is shown, as well as the wide callosal and cingulate sulci. 


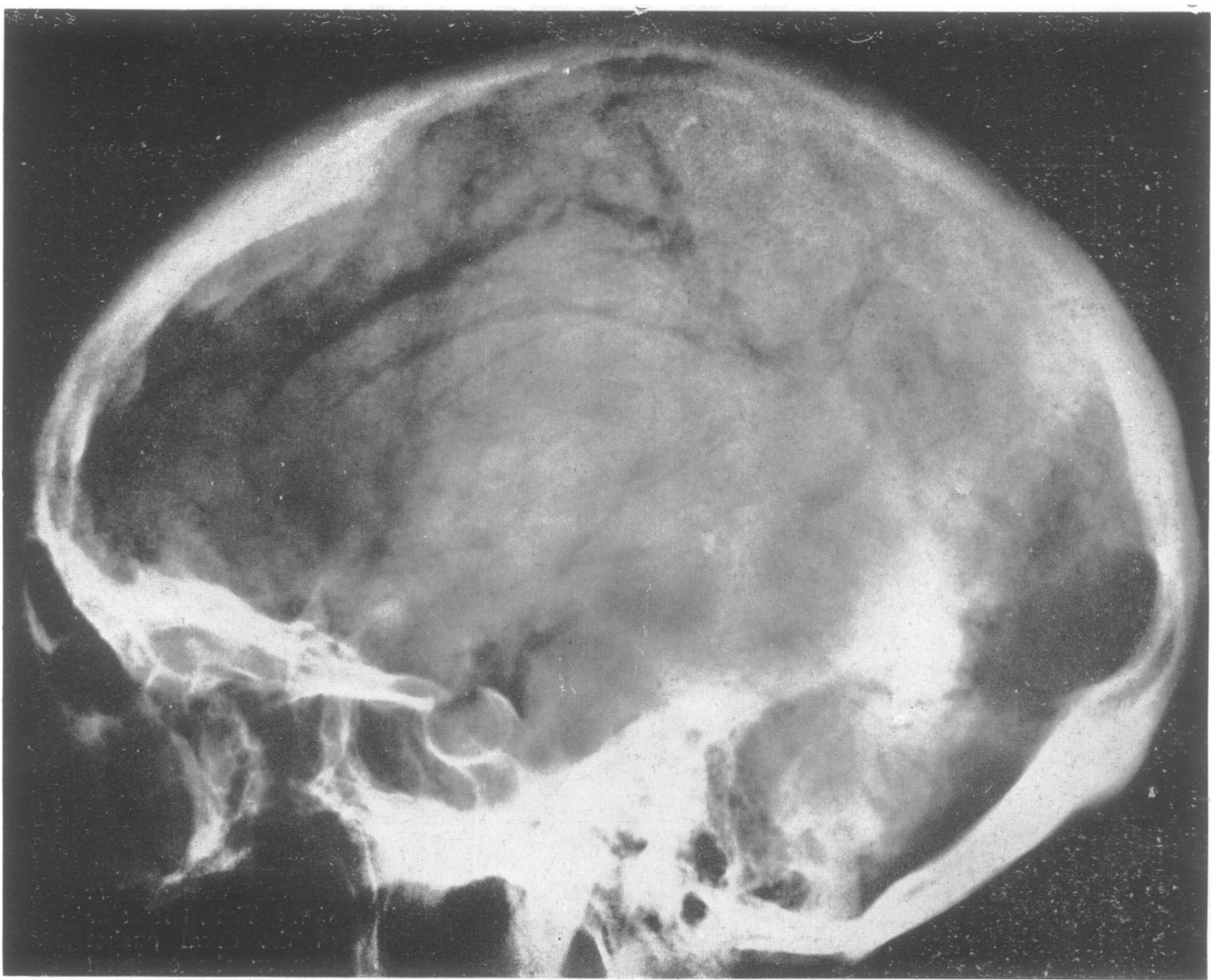

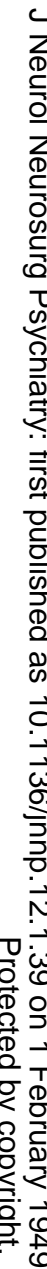

FIG. $8 b$

Fig. 9.-The dura mater seen from behind (Case 3). The occipital poles (over which the dura has been lacerated) are separated by a pocket of dura extending upwards from the posterior fossa.

Fig. 9

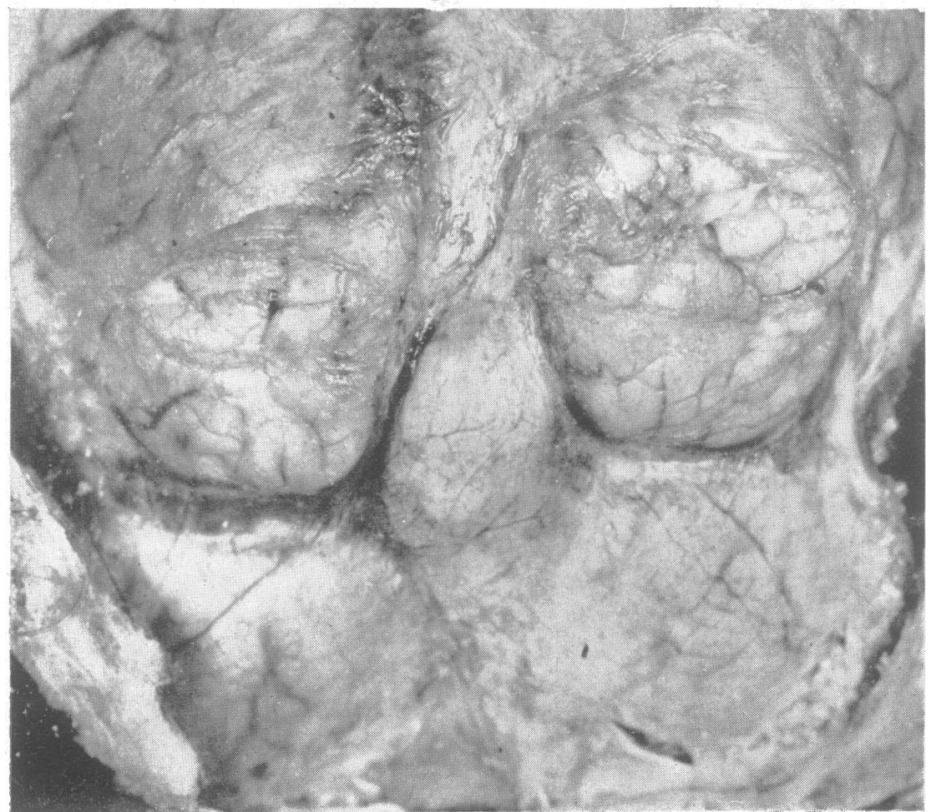



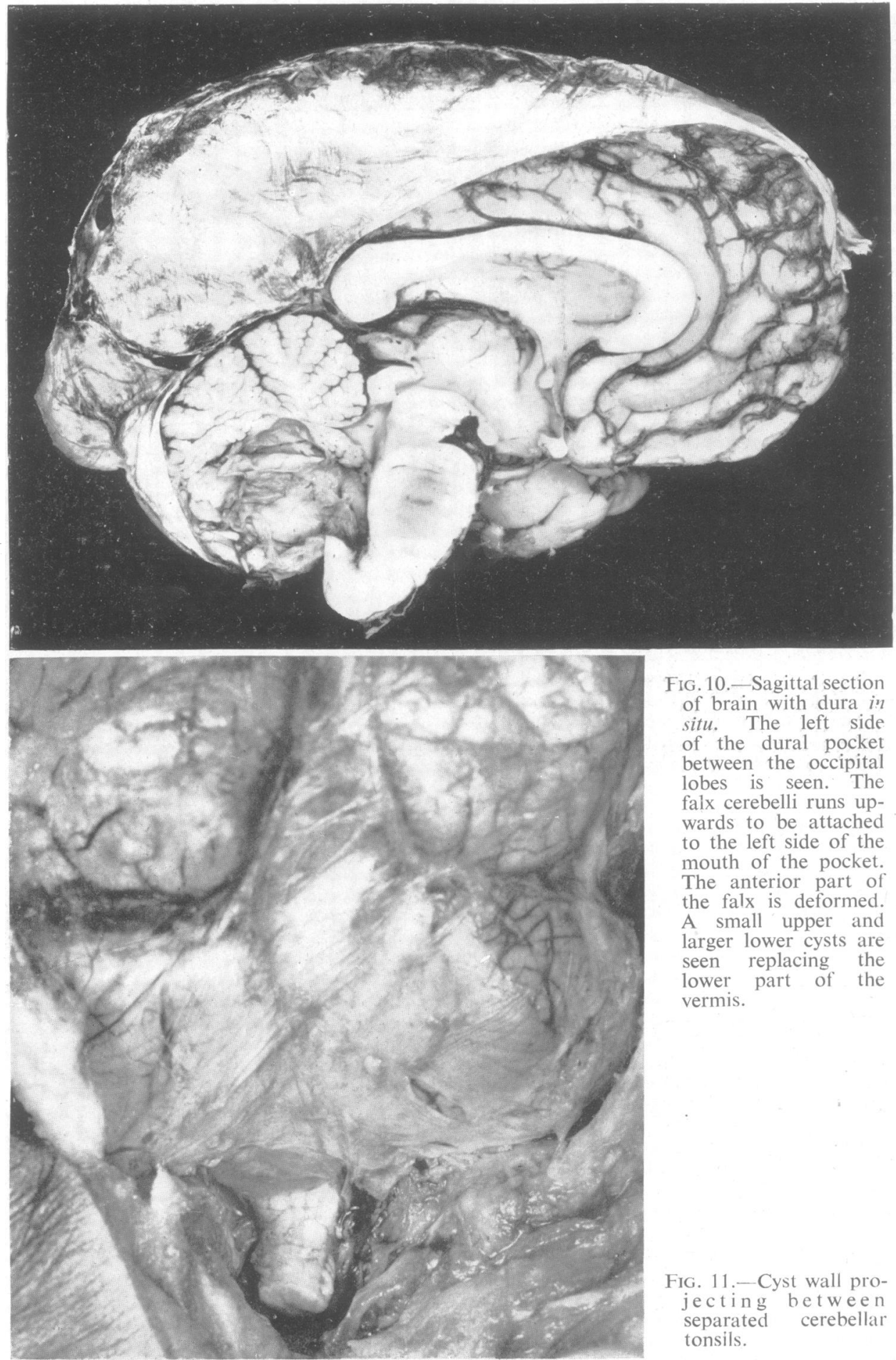

FIG. 10.-Sagittal section of brain with dura $i$ ? situ. The left side of the dural pocket between the occipital lobes is seen. The falx cerebelli runs upwards to be attached to the left side of the mouth of the pocket. The anterior part of the falx is deformed. A small upper and larger lower cysts are seen replacing the lower part of the vermis.

FIG. 11.-Cyst wall projecting between separated cerebellar tonsils. 


\section{DEFECTS OF CISTERNA MAGNA AND DURA MATER}
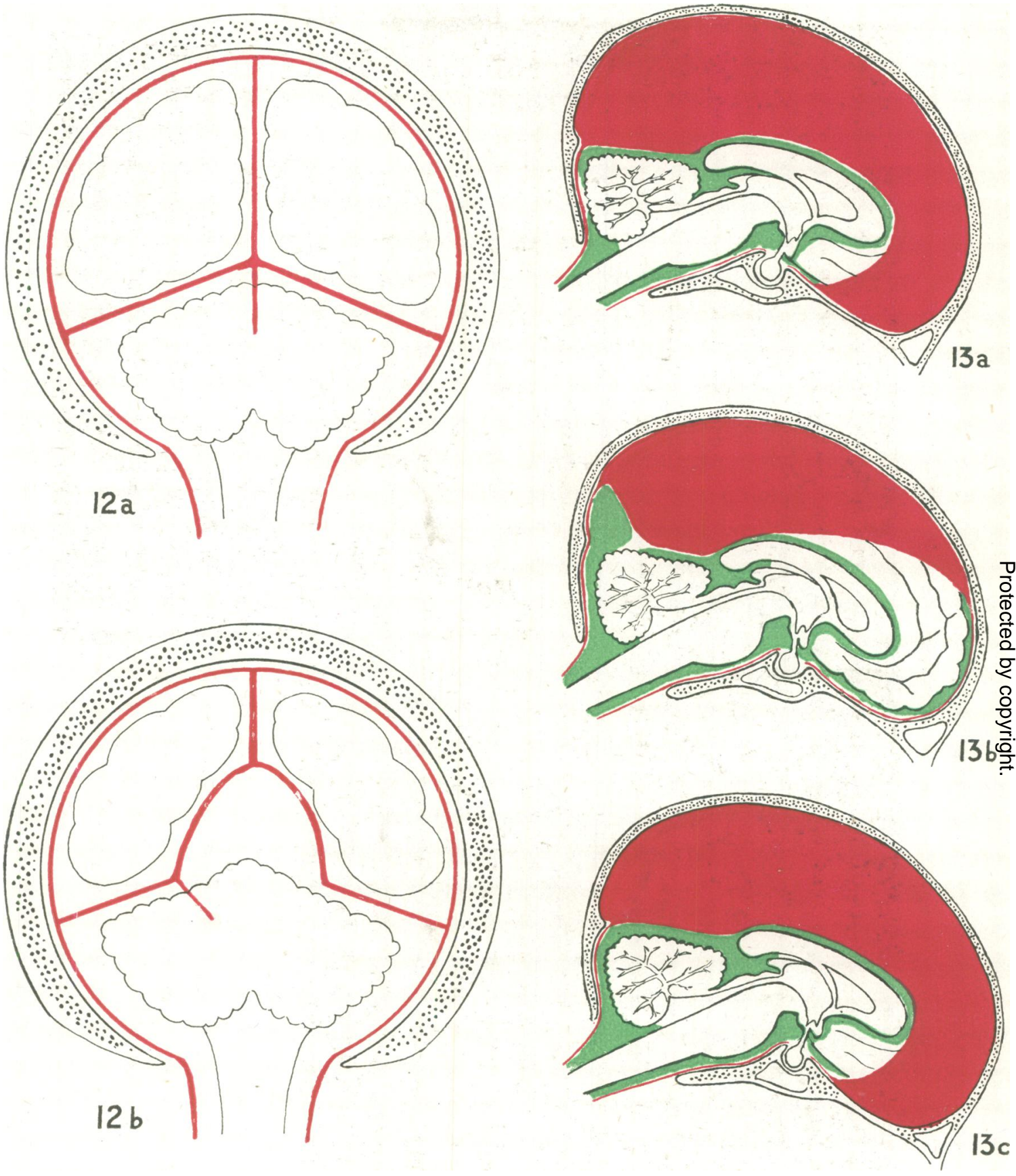

FIG. 12.-Comparison, in coronal sections, of normal and abnormal arrangements of the posterior part of the falx cerebri, the tentorium, and the falx cerebelli.

FIG. 13.-Comparison of normal arrangement of arachnoid and dura, with the abnormality which is the subject of this paper, and with that in which the cisterna magna is not delimited from the remainder of the subarachnoid space over the cerebellum. 
study the dural arrangement which permitted such a surprising appearance.

\section{Allied Abnormalities of Cisterna Magna, Tentorium, and Falx Cerebri \\ CASE RePORT}

A stout woman, 46 years of age, stated that for over twenty years she had suffered from attacks of headache which occurred during the afternoon once every two or three months. It was a severe ache, felt all over the head, and was sometimes associated with vomiting. The headache would disappear after sleep. There were no visual phenomena or bodily dysæsthesiæ.

Twenty months before admission to hospital she began to suffer from prolonged bouts of throbbing pain at the back of her head, which was relieved temporarily by analgesics. About two months later she was sometimes unsteady on her feet, with a variable tendency to stagger to the left side. Five weeks before admission she began to vomit when the headache was severe, on rising from recumbency, or after coughing had induced very severe headache. She was unable to stand unsupported. Lethargy increased to drowsiness, and on several occasions she could not be roused for periods of several hours. At other times she behaved in a peculiar uncontrolled fashion which was referred to as being hysterical.

The only abnormalities detected in the cranial nerves were as follows : upward rotation of the right eye was defective ; conjugate deviation to either side was poorthe right eye adducted poorly and the eyes tended to swing back towards the mid-line; the left corner of the mouth moved slightly less than the right and the uvula was drawn slightly to the left side on articulation. There was no papillodema. The motor power was good. In recumbency there was only slight inco-ordination of movement of all four limbs, with no unilateral preponderance. She would not attempt to walk when assisted into the upright posture, and when supported she tended to fall to the left side whether her. eyes were open or shut. Her head was inclined to the right side, with the chin rotated slightly to the left. The reflexes and sensation were normal.

A diagnosis of neoplasm of the central portion of the cerebellum was made. It was thought probable that it involved the upper part of the vermis, with transmission of pressure to the third nuclei. However, the absence of papillodema at this advanced stage was unexpected, and-chiefly because of this-investigation was undertaken. The pressure of the cerebrospinal fluid was $140 \mathrm{~mm}$. and the response to jugular compression was normal. The fluid was clear and colourless. It contained 1 large mononuclear cell in $3 \mathrm{c} . \mathrm{mm}$. The protein content was $50 \mathrm{mg}$. per $100 \mathrm{c.cm}$. without increase in the globulin fraction. The colloidal gold curve was normal. The Wassermann reaction was normal in blood and cerebrospinal fluid.

Radiographs of the skull disclosed no abnormality. The first postero-anterior film during encephalography showed an unusual collection of gas in the mid-line above the level of the inion (Fig. 8). Lateral films showed it to lie immediately in front of the inner table of the interparietal portion of the occipital bone. The shadow at the level of the inion measured $3.6 \mathrm{~cm}$. in width, and $2 \mathrm{~cm}$. in depth. As filling continued, the collection of gas extended downwards towards the foramen magnum. There was little doubt that this shadow represented the cisterna magna, very much larger than usual and extending above the internal occipital protuberance almost half-way to the lambda, with its apex above the usual level of the tentorium. There appeared to be two possibilities-first, that there was a hiatus in the posterior medial portion of the tentorium through which a cul-de-sac of arachnoid passed before being reflected back on to the cerebellum, or secondly, an abnormal conformation of the dura existed without an actual break in continuity. The posterior surface of the vermis was well outlined and the width of the shadow of gas suggested that the cerebellum was not enlarged. Below, the cistern did not extend forwards to the region of the foramen of Magendie, being interrupted by a globular projection. This extended into the foramen magnum, forming posteriorly an unusually defined and acute angle with the shadow of the inferior surface of the cerebellum. It was taken to represent the depressed cerebellar tonsils. Having filled the cisterna magna, gas began to pass upwards anterior to the medulla and pons into the interpeduncular and ambient cisterns. No manœuvre secured ventricular filling. The pontine and interpeduncular cisterns were small, and the upper surface of the pons appeared to be higher than usual (Fig. 8b). The floor of the third ventricle was slightly depressed. The callosal and cingulate sulci appeared higher than normal, and were wider than usual. It was believed that the appearance suggested the presence of a tumour in the posterior cranial fossa, the absence of ventricular filling being ascribed to blockage of the foramen of Magendie. For a short period the patient's clinical state improved and exploration of the posterior fossa was projected, although it was believed that little prospect of cure existed. However, she passed once again into a drowsy stage which on this occasion deepened to coma, and she died before operation could be performed.

At post-mortem examination the calvarium was separated from the dura and removed. This exposed a globular projection of dura mater between the occipital lobes, the dura being continuous with that over the cerebellar hemispheres below and laterally with that over the occipital lobes (Fig. 9). Veins, larger on the left side, coursed along each side of the convexity, from the longitudinal sinus at its apex to the transverse sinuses below on each side. Dissection showed the bulge to be the posterior wall of a dural pocket, the mouth of which opened below, through a hiatus in the tentorial shelf, into the posterior fossa (Fig. 10). The two lateral walls, meeting anteriorly, appeared to be formed by splitting of the posterior inferior portion of the falx cerebri into two layers which, below, turned sharply laterally to become continuous with the tentorium (Fig. 1 1 b b). The falx cerebelli was well developed. Above, its anterior edge was attached at the meeting point of the tentorium, the falx, and its two derivative layers (Figs. 10 and 12). Behind this point 
it veered to the left, to be attached to the lateral wall of the pocket as it turned to become the tentorial dura. The dural pocket contained a cul-de-sac of arachnoid of the cisterna magna. The posterior part of this membranous pocket was continuous with the arachnoid of the cisterna magna, the anterior wall with that over the superior surface of the cerebellum to which it became firmly applied. The veins which normally meet at the torcula coursed along the angles of the dural sac. The straight sinus divided into three channels, one running upwards to the superior sagittal sinus and one on each side to the transverse sinuses (Fig. 10). The sagittal sinus divided into two main and several smaller trunks which passed downwards to the transverse sinuses (Figs. 9 and 10). The anterior part of the falx cerebri was also malformed. Its anterior edge passed forwards and upwards, above the splenium of the corpus callosum, to reach the dura over the cerebral hemispheres above the genu of the corpus callosum (Fig. 10). There was no dura between the medial surfaces of the frontal lobes.

Thus, the dural membrane shows two unusual anomalies together with a very large cisterna magna. Fig. 13 illustrates diagrammatically the normal and abnormal arrangements of the dura and cisterna magna, and shows also the anomaly previously described in which absence of occlusion of the upper part of the cisterna magna allows gas to rise directly to the superior surface of the cerebellum (see Fig. 4). Although pathological confirmation is lacking, it is probable that a similar defect exists in the posterior part of the dura whenever the cisterna magna extends above the inion, as in the two further cases mentioned. It is not known whether the anteriorly placed defect was present in the other instances. It is possible that the dural defect might exist without abnormality of the cisterna magna, in which case the anomaly would not be recognized during encephalography.

The dural pocket and neighbouring occipital lobes have a stable appearance which suggested a life-long existence. Microscopically, the membrane resembled normal dura although it contained a number of dilated venules, the distension being probably due to terminal venous congestion. There were no indications of preceding inflammation in the sections. Thus, a developmental lesion seems to be the most likely explanation. If this was due to maldevelopment, two possibilities arise : injury (inflammatory, vascular or traumatic) with subsequent repair causing an unusual pattern; or genetic influences. It is possible that future observations may provide evidence to decide which is the cause ; for example, the presence of a familial incidence would favour a genetic cause.

\section{Account of Coincident Cyst in Vermis}

When, during removal, the dura and arachnoid were incised transversely at the level of the foramen magnum, a translucent membrane, enclosing clear fluid, was seen to project between the cerebellaf tonsils (Fig. 11). There were no adhesions between this membrane and the overlying arachnoid Median section of the brain showed it to be parf of the thin wall of the lower of two cysts which occupied most of the lower part of the vermis an pressed into the lateral lobes of the cerebellum on each side (Fig. 10). The upper cyst reached the medullary body of the cerebellum above, and: anteriorly was separated from the roof of the loween? part of the fourth ventricle by a thin layer of cerebellar tissue apparently representing the nodule? Posteriorly the tuber and pyramid of the vermis were seen and appeared to be compressed. The uvula had largely disappeared, being representedw only by a little tissue. The foramen of Magendiewas patent and higher than normal, at much the same level as the double wall between the two cysts $\vec{\omega}$ Small vascular tufts, continuous with the choroide plexus of the fourth ventricle, ran into small vessels which dispersed over the anterior upper part of the wall of the lower loculus. The arachnoid of thẹ cisterna magna was not connected with the cyst. It was apparent that the shadow in the encepha和 grams which was taken to represent the cereberas? tonsils was, in reality, due to the lower part of cyst projecting between the cerebellar tonsils. The wall was composed of a thin layer of delicate fibrofis tissue with an internal lining of a single layer cuboidal cells. Some small vessels ran between cyst wall and the atrophied cerebellar tissue in $\overrightarrow{4}+\overrightarrow{0}$ neighbourhood. Neurons and glia were seen in the remnants of vermis applied to the wall of the cyst. The cuboidal cells of the walls of the cyse were similar in appearance to normal ependyma cells. There was no arachnoidal thickening, and the cyst was not connected with the arachnoidळ The microscopical appearance, the position of the cyst, the absence of arachnoidal abnormality, and the relationship of the choroid plexus, raises the possibility of an ependymal origin. Such cysts are rare, but a number having a similar position have been described, notably by Zehnder (1938). He believed the cysts to be similar in type and origin to others which he described in the Sylvian fissure and between the cerebral hemispheres, and ascribed an arachnoidal origin. Most were lined by single layer of cuboidal cells, but one had severa? layers of cells with an internal layer of pigment? storing ciliated epithelium.

Structurally, these cysts are unlike the condition described by Horrax (1924) under the titles "Generalized Cisternal Arachnoiditis," although each may produce " the localizing features of cerebellar lesion simulating tumour." 'In the lattef 
group of cases there was " arachnoid thickening of the basilar cisternæ às well as that overlying the cerebellum." Operative exploration disclosed a greatly dilated posterior cistern with thickened arachnoid membrane containing pent-up cerebrospinal fluid. The foramen of Magendie was blocked, causing internal hydrocephalus. The condition was believed to be an inflammatory one.

Allen and Corkill (1937) described a number of cases in which arachnoidal cysts occupied the cisterna magna. Such deep penetration of the cerebellum was not apparent in their cases as in that pictured by Zehnder, and that here described. Their cases appeared to be similar to those described by Horrax.

Shryock and Alexander (1943) reported the clinical and pathological findings in a fourteen year old girl who had a number of developmental anomalies. There was evidence of mild cerebellar dysfunction eight months before her death from peritonitis following volvulus. They describe the fourth ventricle as being enlarged at the expense of the substance of the cerebellar vermis and continuous with a cyst which extended caudad between the cerebellar tonsils and laterally on each side of the medulla oblongata. The cyst was bounded by a membrane which was continuous with the ependyma of the rhomboid fossa internally, and with the arachnoid covering the cerebellum externally. Microscopically the lining consisted of ependyma and nervous tissue. There were two small, laterally placed cysts, " the significance of which was not clear." They collected eight other cases in the literature, mostly young, but one aged 59 years. Five of the eight cases presented symptoms suggesting cerebellar disease. Nystagmus was noted in three cases, positive Rhomberg's sign in two, and hypotonia in one. Gradual increase in the size of the head was mentioned in four instances. Enlargement of the third and lateral ventricles was noted in five cases, of the third alone in another. In three, the foramina of Luschka and Magendie were obstructed, in one the foramina of Luschka were patent. Four died following surgery. There was considerable variation in the degree of deformity of the cerebellar vermis. Some authors thought that parts of the vermis had disappeared, others that all parts were present, even if rudimentary.

Following Dandy they believe the condition to be due to obstruction to the foramina of Magendie and Luschka by inflammatory processes, or to their failure to develop; or perhaps to tardy opening of the foramina. This is thought to lead to dilatation of the fourth ventricle, which during the fourth month of intra-uterine life encroaches upon the embryonic structures which are fusing to form the posterior portion of the cerebellar vermis, causing them to remain rudimentary. Opening of the foramina might occur at a later time, allowing circulation of the cerebrospinal fluid and cessation of dilatation.

A case fully reported by Sahs (1941) and more clearly described than most of the other pathological conditions in this region is included in Shryock and Alexander's collected series. This case is much more suggestive of agenesis of the vermis without primary obstruction to the foramina of Magendie and Luschka. Had the anterior wall of the cyst disappeared in the case recorded here, the appearance would have been very similar to that described by Shryock and Alexander. In this case the association with other developmental defects of the dura favours a developmental cause for the cyst in the posterior fossa. However, the advanced age at which symptoms appeared suggests that the cyst developed late in life, although its seeds might have been due to factors active during intra-uterine growth.

Thus inflammatory, traumatic, and congenital causes have been evoked to explain the presence of cysts presenting in the cisterna magna, some associated with atrophy of the vermis. It is probable that there is more than one type of cyst presenting in this region. Cysts replacing the lower median part of the vermis may arise from ependyma, perhaps during intra-uterine growth, perhaps later in life, and they probably constitute a different group from the arachnoidal cysts which commence in the cisterna magna. Disappearance of parts of the cyst wall would result in an appearance similar to that which has been thought to be due to maldevelopment of the vermis, but it is not known whether this occurs.

\section{Summary}

The normal arrangement of the cisterna magna is déscribed.

Four variations are recorded which owe their recognition to encephalography. Each calls for modification of encephalographic technique if ventricular filling is to be attained.

A large cisterna magna, seen during encephalography to extend above the inion, was associated with abnormalities of falx cerebri and tentorium, which are considered to be of developmental origin.

REFERENCES
Allen, I. M., and Corkill, H. K. (1937). New Zealand
med. J., 36, 291.

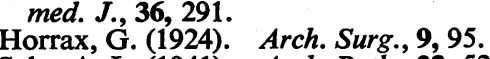

Sahs, A. L. (1941). $\quad$ Arch. Path., 32, 52.

Shryock, E. H., and Alexander, H. B. (1943). Bull. Los. Angeles Neurol. Soc., 8, 11.

Zehnder, M. (1938). ZZbl. Neurochirurgie, 3, 100. 